\title{
gु \\ Bismuth in strong magnetic fields: Unconventional Zeeman coupling and correlation effects
}

\author{
Jason Alicea ${ }^{1}$ and Leon Balents ${ }^{2}$ \\ ${ }^{1}$ Department of Physics, California Institute of Technology, Pasadena, California 91125, USA \\ ${ }^{2}$ Kavli Institute for Theoretical Physics, University of California, Santa Barbara, California 93106-9530, USA
}

(Received 27 October 2008; revised manuscript received 16 April 2009; published 1 June 2009)

\begin{abstract}
While the behavior of strongly interacting two-dimensional electrons in high magnetic fields is by now well understood, our understanding of the three-dimensional (3D) case is comparatively rudimentary. Illuminating this disparity are recent experiments on 3D bismuth, where unanticipated transport and magnetization structure-including hysteresis-persist even when all carriers are expected to reside in the lowest Landau level. Motivated by these findings, we derive a low-energy Hamiltonian for the hole and three Dirac electron pockets in bismuth which, crucially, encodes an unconventional Zeeman effect generated by spin-orbit coupling. We show that (1) this Zeeman coupling strongly suppresses the quantum limit for the Dirac electrons, giving rise to the observed magnetization structure, and (2) the hysteresis coincides with one of the pockets emptying its second Landau level, which is where Coulomb effects are most pronounced. Incorporating interactions, we find instabilities toward charge-density-wave and Wigner crystal phases and propose that hysteresis arises from a first-order transition out of the latter.
\end{abstract}

DOI: 10.1103/PhysRevB.79.241101

After more than a century of active research, bismuth continues to yield fascinating discoveries. Much of this material's exceptional behavior stems from its band structure ${ }^{1}-$ the Fermi surface arises from a hole pocket and three Dirac electron pockets which contribute an extremely low carrier density of $3 \times 10^{17} \mathrm{~cm}^{-3}$. An important consequence of the small carrier concentration is that the quantum limit, at which carriers are confined to the lowest Landau level (LL) (LLL), can be realized with laboratory fields. (Conventional metals, by contrast, are typically well described semiclassically even at the largest accessible fields.) The interplay between quantum mechanics and interactions in the quantum limit generates highly exotic behavior in two dimensions but is relatively poorly understood in three dimensions (3D). This fundamental issue is becoming more pressing due to recent experimental progress in bismuth. ${ }^{2-4}$

For fields along the highest-symmetry "trigonal" axis, the hole quantum limit in bismuth occurs at $B \sim 9 \mathrm{~T} .5$. The electron quantum limit, while less clear experimentally, is believed to occur at similar fields. ${ }^{3}$ From a single-particle perspective, quantum oscillations should subside once all carriers reside in the LLL, and transport and thermodynamic quantities should appear "featureless." Bismuth nevertheless exhibits surprisingly rich physics far beyond $9 \mathrm{~T}$. Nernst peaks were resolved at 13.3, 22.3, and $30.8 \mathrm{~T}$, with the Hall resistance exhibiting steplike features in between, prompting the suggestion that fractionalization may occur in this 3D system. ${ }^{2}$ Similar anomalies appear in bismuth-antimony alloys. ${ }^{4}$ More recent torque magnetometry studies at fields near the trigonal axis additionally measured unanticipated magnetization structure-including hysteresis-persisting up to $31 \mathrm{~T}$, providing further evidence for correlation physics. ${ }^{3}$

These puzzling observations call for a renewed look at bismuth's LL spectrum as well as Coulomb-driven instabilities. Here, we derive a low-energy theory for bismuth suitable for addressing these issues. We first show that strong atomic spin-orbit coupling generates an unconventional Zeeman effect for the Dirac electrons that is absent from the Cohen-Blount model $^{6}$ and suppresses their quantum limit far
PACS number(s): 71.45.Lr, 71.10.Pm, 72.20.Ht, 71.70.Ej

beyond $9 \mathrm{~T}$ for trigonal fields. Thus, quantum oscillations persist to much larger fields than has been assumed, which explains quantitatively the high-field magnetization structure observed in Ref. 3 [see Fig. 1(a)]. However, the hysteresiswhich we show occurs when one of the electron pockets empties its second LL-likely has a many-body origin. To address this aspect of experiment, we study interaction effects near this band emptying, employing functional renormalization-group (FRG) techniques similar to Ref. 7 and going beyond Abrikosov's early analysis. ${ }^{8}$ As the Fermi energy passes from above the second LL into the LLL, we find that the leading instability for these electrons involves charge-density-wave (CDW) order in the second LL, followed by Wigner crystal formation, and LLL CDW order [see Fig. 1(c)]. We suggest that the hysteresis originates from the Wigner crystal phase and discuss experiments to verify this proposal. Finally, we comment briefly on the Nernst- and Hall-effect puzzles, which remain unexplained by this work.

Our starting point is the tight-binding model of Liu and Allen, ${ }^{9}$ which was constructed to accurately reproduce bismuth's band structure near the Fermi level. ${ }^{1}$ As in graphene, it will prove extremely useful to derive from this microscopic model an effective low-energy Hamiltonian for the electrons and holes in a magnetic field $\mathbf{B}$ by expanding the lattice fermion operators as follows:

$$
f_{\mu j \alpha}(\mathbf{r}) \sim e^{i \mathbf{Q} \cdot \mathbf{r}} \varphi_{\mu j \alpha}^{\beta} h_{\beta}^{\dagger}+\sum_{\lambda=1}^{3} e^{i \mathbf{P}_{\lambda} \cdot \mathbf{r}} \sum_{\ell, a=1}^{2} \phi_{\mu j \alpha}^{\lambda \ell a} \psi_{\lambda \ell a},
$$

where $\mu=s, p_{x, y, z}$ labels the outer-shell orbital with spin $\alpha$ $=\uparrow / \downarrow$ at site $\mathbf{r}$ on sublattice $j=1,2$. The two-component operators $h$ describe hole excitations near the $T$ point $\mathbf{Q}$, and the four-component Dirac fermions $\psi_{\lambda}$ describe electron excitations near the $L$ points $\mathbf{P}_{\lambda}$; the corresponding wave functions are $\varphi^{\beta}$ and $\phi^{\lambda \ell a}$. Using Eq. (1), we obtain the noninteracting Hamiltonian $H_{0}=\int_{\mathbf{r}}\left(h^{\dagger} \mathcal{H}_{h} h+\Sigma_{\lambda} \psi_{\lambda}^{\dagger} \mathcal{H}_{e}^{\lambda} \psi_{\lambda}\right)$,

$$
\mathcal{H}_{h}=-\mu_{h}-\frac{D_{x}^{2}+D_{y}^{2}}{2 m_{\perp}}-\frac{D_{z}^{2}}{2 m_{z}}-\frac{g_{h} \mu_{B} B^{z} \sigma^{z}}{2},
$$



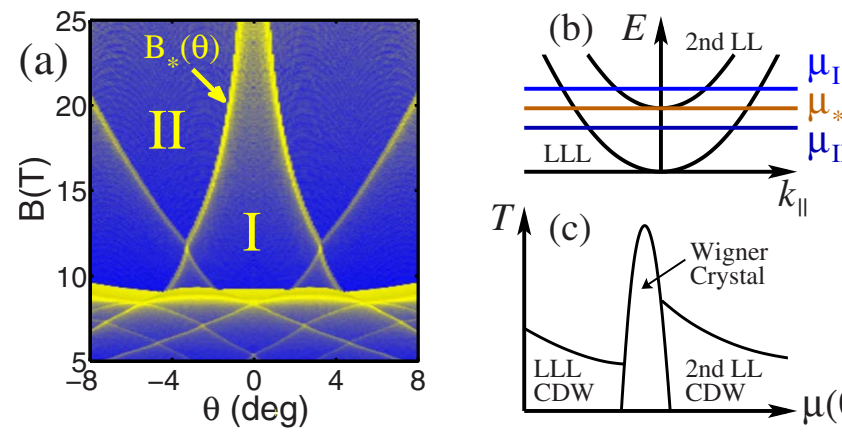

FIG. 1. (Color online) (a) Single-particle DOS, excluding the contribution from the electron pocket invisible in torque experiments (Ref. 3). All electron pockets occupy the second LL in I, while pocket 3 empties into the LLL in II. (b) Schematic energy dispersion for pocket 3 and (c) proposed phase diagram near $B_{*}(\theta)$.

$$
\mathcal{H}_{e}^{3}=-\mu_{e}+m \mu^{z}-i \sum_{j=x, y, z} v_{j} D_{j} \eta^{j}-\frac{\mu_{B} \mathbf{G} \cdot \mathbf{B}}{2} .
$$

Here $\mathbf{D}=\nabla-i q \mathbf{A}$, with $\mathbf{B}=\nabla \times \mathbf{A}$ and $q= \pm e$ for the holes and electrons, respectively. We employ three sets of Pauli matrices via $\sigma_{\alpha \beta}^{j} h_{\beta}, \tau_{\ell \ell^{\prime}}^{j} \psi_{\lambda \ell^{\prime} a}$, and $\mu_{a b}^{j} \psi_{\lambda \ell b}$ above and define $\eta^{x}=\mu^{x} \tau^{z}$ and $\eta^{y, z}=\left(v_{1 y, z} \mu^{y}+v_{2 y, z} \tau^{x} \mu^{x}\right) / v_{y, z}$, where $v_{1 j}^{2}+v_{2 j}^{2}$ $=v_{j}^{2}$. The Hamiltonians $\mathcal{H}_{e}^{1,2}$ for pockets 1 and 2 can be obtained from $\mathcal{H}_{e}^{3}$ by rotating $\mathbf{D}, \mathbf{B}$ by $\pm 2 \pi / 3$ about the trigonal $(z)$ axis. In terms of the electron mass $m_{e}$ and speed of light $c$, we have $\mu_{e}=0.0335 \mathrm{eV}, \mu_{h}=0.012 \mathrm{eV}, m_{\perp}$ $=0.0675 m_{e}, m_{z}=0.612 m_{e}, m=6 \mathrm{meV}, v_{x}=0.003 c, v_{1 y}=$ $-7.6 \times 10^{-5} c, \quad v_{2 y}=3.4 \times 10^{-4} c, \quad v_{1 z}=0.002 c, \quad$ and $v_{2 z}=$ $-0.0014 c$. The Fermi energy $E_{F}=0$ when $\mathbf{B}=0$ but changes to maintain charge neutrality when $\mathbf{B} \neq 0$.

Zeeman coupling warrants separate discussion. Microscopically, Zeeman energy has one source $\propto \mathbf{B} \cdot(\mathbf{L}+2 \mathbf{S})$ and another coming from spin-orbit coupling $\propto(\nabla U \times \mathbf{A}) \cdot \mathbf{S}$, where $U$ is the crystal potential and $\mathbf{L}, \mathbf{S}$ denote orbital/spin angular momentum. While the corresponding low-energy terms can be obtained via Eq. (1), evaluating the spin-orbit contribution is nontrivial since the potential $U$ is unknown. Progress can be made, however, by assuming $U$ is rotationally invariant, as then only two independent matrix elements remain: $\chi_{s / p}=\frac{1}{4 m_{e} c^{2}}\left\langle s / p_{x} \uparrow\left|r \partial_{r} U \sin ^{2} \theta\right| s / p_{x} \uparrow\right\rangle$, where $\left|s / p_{x} \uparrow\right\rangle$ are the atomic $s / p_{x}$ orbital wave functions. With this assumption, we obtain the hole Zeeman splitting in Eq. (2), which is sensitive only to $B^{z}$, consistent with experiment. ${ }^{5}$ For the electrons, we obtain the coupling in Eq. (3) with $G_{x}=\tau^{x}\left(g_{1 x}\right.$ $\left.+g_{2 x} \mu^{z}\right)$ and $G_{y, z}=\tau^{y}\left(g_{1 y, z}+g_{2 y, z} \mu^{z}\right)+\tau^{z}\left(g_{3 y, z}+g_{4 y, z} \mu^{z}\right)$. The $g$ factors are listed in Table I. Crucially, $g_{h}$ depends only on $\chi_{p}$, and the electron $g$ factors obtain only a weak contribution from $\chi_{s}$ because the wave functions $\phi^{\lambda \ell a}$ are concentrated on the $p$ orbitals. The value of $\chi_{s}$ is thus unimportant, and we will simply set $\chi_{s}=\chi_{p}$. Finally, the hole Zeeman splitting has been well studied experimentally, ${ }^{10}$ and from the available data we deduce that $g_{h} \approx 54$, which allows us to determine $\chi_{p}$ and the electron $g$ factors. ${ }^{11}$

Equations (2) and (3) constitute our first major result. Most importantly, the electron Zeeman coupling has not been considered previously and modifies the spectrum dramatically at high fields as we now discuss.

While the hole Hamiltonian is easily diagonalized, the electron part is nontrivial since the Zeeman and orbital terms do not commute. To proceed, we truncate the Hilbert space, including only the first $n \sim 10 \mathrm{LLs}$, and diagonalize the Hamiltonian numerically. Motivated by Ref. 3, we study the spectrum for fields tilted by an angle $\theta$ from the trigonal toward the binary $(x)$ axis. Figure 1(a) displays the singleparticle density of states (DOS) in the $B-\theta$ plane (excluding the electron pocket invisible to experiments $\left.{ }^{3}\right)$. Bright lines occur where the Fermi energy crosses the bottom of a LL, and the flat line at $\sim 9 \mathrm{~T}$ corresponds to the hole quantum limit. Remarkably, the electrons give rise to features at much higher fields which agree well with the anomalous structure reported experimentally (see Fig. 3 in Ref. 3).

These features actually reflect quantum oscillations that arise from an increase in carrier density with field ${ }^{5}$ and modifications of the electron spectrum by Zeeman coupling. Without Zeeman, the LLL energies are field independent, and all other LLs are doubly degenerate (see, e.g., Ref. 3), similar to the situation in graphene. Zeeman coupling mixes these LLs, leading to an increase in the LLL energies and a splitting of the higher-LL degeneracy. This diminishes the separation between the LLL and second LL and strongly suppresses the electron quantum limit. Indeed, in region I of Fig. 1(a) all electron pockets occupy the second LL. Tilting the field into region II pushes one of those pockets (assumed to be pocket 3 hereafter) into the LLL. The dispersion for pocket 3 versus the momentum $k_{\|}$along the field appears schematically in Fig. 1(b), together with the chemical potential in I and II. Interestingly, experiments observed hysteresis in the magnetization at the line labeled $B_{*}(\theta)$ separating these regions [corresponding to $\mu_{*}$ in Fig. 1(b)]. Addressing this puzzle requires moving beyond single-particle physics.

We now incorporate Coulomb interactions,

TABLE I. Electron/hole $g$ factors, with $g_{\alpha}=a_{\alpha}+b_{\alpha} \chi_{p}+c_{\alpha} \chi_{s}$.

\begin{tabular}{lcccccrc}
\hline \hline & $a$ & $b$ & $c$ & & $a$ & \multicolumn{1}{c}{$b$} & $c$ \\
\hline$g_{h}$ & 4 & 2 & 0 & $g_{4 y}$ & -0.90 & -0.33 & 0.049 \\
$g_{1 x}$ & 1.5 & 1.3 & 0.072 & $g_{1 z}$ & -0.082 & 0.19 & 0.030 \\
$g_{2 x}$ & -1.4 & -0.44 & -0.024 & $g_{2 z}$ & -0.44 & -0.45 & -0.050 \\
$g_{1 y}$ & 0.88 & 0.32 & -0.0042 & $g_{3 z}$ & 0.36 & 0.42 & -0.0050 \\
$g_{2 y}$ & 0.95 & 0.77 & 0.049 & $g_{4 z}$ & 0.51 & 0.11 & 0.049 \\
$g_{3 y}$ & -1.2 & -0.93 & -0.030 & & & & \\
\hline \hline
\end{tabular}




$$
H_{\text {int }}=\frac{1}{2} \int_{\mathbf{r r}^{\prime}} V\left(\mathbf{r}-\mathbf{r}^{\prime}\right) \rho(\mathbf{r}) \rho\left(\mathbf{r}^{\prime}\right),
$$

where $\rho=\Sigma_{\lambda} \psi_{\lambda}^{\dagger} \psi_{\lambda}-h^{\dagger} h$ and $V(\mathbf{r})$ is the screened Coulomb potential. Interactions can be most simply treated at weak coupling, which is controlled provided the "fine-structure constants" satisfy $e^{2} / v_{a}^{n} \ll 1$, where $v_{a}^{n}$ is the Fermi velocity for pocket $a$ in LL $n$. This criterion inevitably breaks down near a band emptying since at least one $v_{a}^{n} \rightarrow 0$. Correlation effects will be most pronounced in this strongly coupled regime, and this is indeed where hysteresis appears. Our aim now is to study the crossover from weak to strong coupling to understand this aspect of experiment. Rather than addressing the fate of all carriers, we will focus on the leading instability involving pocket 3 electrons near $B_{*}(\theta)$.

Even this restricted question requires considering numerous interactions since $H_{\text {int }}$ couples pocket 3 to all other carriers, some of which occupy more than one LL. We can, however, further distill the problem using an intuitive principle. Similar carriers generally couple more effectively than dissimilar ones. For instance, velocity anisotropies sharply distort the LL wave functions at each electron pocket, making it difficult for interpocket correlations to develop. Such "wave-function mismatch" along with large differences between the electron and hole Fermi velocities similarly disfavors "excitonic" electron-hole pairing instabilities. ${ }^{12}$ We will thus focus on interactions involving only pocket 3 electrons.

Before turning to the details, we provide a simple physical picture for our results. In region II where pocket 3 is confined to the LLL, the perfect Fermi-surface nesting naturally leads to a LLL CDW instability, ${ }^{13}$ where the system gains exchange energy by modulating the density along the field direction. When the chemical potential first crosses the line $B_{*}(\theta)$, the dilute second LL electrons are inherently strongly interacting as noted above. Crudely, there the second LL kinetic energy becomes closer to a flat band, and interactions therefore Wigner crystallize these carriers. Since interactions can scatter two LLL electrons into the second LL and back, the LLL also "inherits" some of this strong correlation, resulting in a simultaneous enhancement of the LLL CDW instability. As the second LL populates further in region I, the system eventually re-enters the weak-coupling regime, and the Wigner crystal is replaced by a second LL CDW.

We flesh out this physical picture formally by employing the FRG method, which gives the renormalized interactions at length scale $L$ as a function of the rescaling factor $\ell$ $=\ln (L / \lambda)$, with $\lambda$ as a microscopic length on the order of the Fermi wavelength. We employ Landau gauge, where the LL wave functions are labeled by the transverse momentum $k_{\perp}$, and set $\ell_{B}=1$. Transverse momentum dependence in the potential $V(\mathbf{q})$ will also be ignored, which allows us to scale away the velocity anisotropy in pocket 3 .

Consider region II first. Exploiting the similarity to a onedimensional metal, we focus on still smaller energy scales by linearizing the kinetic energy around the Fermi momenta $\pm k_{F 3}^{0}$ and expanding $\psi_{3}$ in terms of right/left movers. Projecting onto the LLL, the kinetic energy is $H_{K E}^{3 \text {,II }}$ $=\int_{\mathbf{k}} v_{3}^{0} k_{\|}\left[c_{3 R}^{0 \dagger}(\mathbf{k}) c_{3 R}^{0}(\mathbf{k})-c_{3 L}^{0 \dagger}(\mathbf{k}) c_{3 L}^{0}(\mathbf{k})\right]$, while interactions read

$$
\begin{aligned}
H_{\mathrm{int}}^{3, \mathrm{II}}= & \int_{\mathbf{k}_{i}} \rho\left(k_{\perp 1}, k_{\perp 2}\right) c_{3 R}^{0 \dagger}\left(\mathbf{k}_{1}+\mathbf{k}_{3}\right) c_{3 L}^{0 \dagger}\left(\mathbf{k}_{2}+\mathbf{k}_{3}\right) c_{3 L}^{0}\left(\mathbf{k}_{3}\right) \\
& \times c_{3 R}^{0}\left(\mathbf{k}_{1}+\mathbf{k}_{2}+\mathbf{k}_{3}\right) .
\end{aligned}
$$

Here $\mathbf{k}=\left(k_{\perp}, k_{\|}\right)$and $c_{3 R / L}^{0 \dagger}$ creates a right-/left-moving LLL electron. The one-loop FRG equation for the coupling $\rho(k, x) \equiv \rho(\mathbf{r})$ Fourier transformed in the second argument is

$$
\partial_{\ell} \rho(\mathbf{r})=\frac{1}{2 \pi v_{3}^{0}}\left[\rho(\mathbf{r})^{2}-(\rho * \rho)(\mathbf{r})\right],
$$

with $(f * g)(\mathbf{r})=\int_{\mathbf{r}^{\prime} \mathbf{r}^{\prime \prime}} e^{i\left[\mathbf{r} \wedge \mathbf{r}^{\prime}+\mathbf{r}^{\prime} \wedge \mathbf{r}^{\prime \prime}+\mathbf{r}^{\prime \prime} \wedge \mathbf{r}\right]} f\left(\mathbf{r}^{\prime}\right) g\left(\mathbf{r}^{\prime \prime}\right)$. At the initial conditions, $\rho(r ; \ell=0) \approx g e^{-r^{2} / 2}$, where $g>0$. An approximate solution can be obtained ${ }^{7,14}$ by neglecting the $(\rho * \rho)$ term, which yields $\rho(r ; \ell)=\left[\rho(r ; 0)^{-1}-\ell /\left(2 \pi v_{3}^{0}\right)\right]^{-1}$. Divergence of $\rho(r=0)$ at $\ell^{*}=2 \pi v_{3}^{0} / g$ triggers an instability where $\left\langle c_{3 R}^{0 \dagger} c_{3 L}^{0}\right\rangle$ condenses, corresponding to $2 k_{F 3}^{0} \mathrm{CDW}$ formation along the field with uniform transverse density. The neglected $(\rho * \rho)$ term merely reduces the transition temperature. ${ }^{7}$

Next, consider region I, with the chemical potential slightly above the second LL. Here we derive a theory for right/left movers in the LLL $c_{3 R / L}^{0}$ and second LL $c_{3 R / L}^{1}$ Pocket 3 interactions now involve several couplings,

$$
\begin{aligned}
H_{\mathrm{int}}^{3, \mathrm{I}}= & \int_{\mathbf{k}_{i}}\left\{\rho\left(k_{\perp 1}, k_{\perp 2}\right) c_{3 R}^{0 \dagger}\left(\mathbf{k}_{1}+\mathbf{k}_{3}\right) c_{3 L}^{0 \dagger}\left(\mathbf{k}_{2}+\mathbf{k}_{3}\right) c_{3 L}^{0}\left(\mathbf{k}_{3}\right)\right. \\
& \times c_{3 R}^{0}\left(\mathbf{k}_{1}+\mathbf{k}_{2}+\mathbf{k}_{3}\right)+u c_{3 R}^{1 \dagger} c_{3 L}^{1 \dagger} c_{3 L}^{1} c_{3 R}^{1} \\
& +\left[v c_{3 R}^{0 \dagger} c_{3 L}^{1 \dagger} c_{3 L}^{1} c_{3 R}^{0}+(R \rightarrow L)\right] \\
& \left.+\left[w c_{3 R}^{0 \dagger} c_{3 L}^{0 \dagger} c_{3 L}^{1} c_{3 R}^{1}+\text { H.c. }\right]\right\}
\end{aligned}
$$

where the arguments of the last three couplings should appear as in the first. Fourier transforming in the second argument and defining $\mathbf{r}=(k, x)$ as before, it will be convenient below to write the FRG equations as follows:

$$
\begin{gathered}
\partial_{\ell} \rho(\mathbf{r})=\frac{1}{2 \pi v_{3}^{0}}\left[\rho(\mathbf{r})^{2}-(\rho * \rho)(\mathbf{r})\right]-\frac{\alpha}{\pi}\left(w * w^{*}\right)(\mathbf{r}), \\
\partial_{\ell} u(\mathbf{r})=\epsilon u(\mathbf{r})+\frac{\beta}{\pi} u(\mathbf{r})^{2}-\frac{\alpha}{\pi}(u * u)(\mathbf{r})-\frac{\left(w^{*} * w\right)(\mathbf{r})}{2 \pi v_{3}^{0}}, \\
\partial_{\ell} v(\mathbf{r})=\frac{\gamma_{1}}{2 \pi}\left[v(\mathbf{r})^{2}-(v * v)(\mathbf{r})\right]+\frac{2 \gamma_{2}}{\pi}|w(\mathbf{r})|^{2}, \\
\partial_{\ell} w(\mathbf{r})=\frac{\epsilon}{2} w(\mathbf{r})-\frac{1}{2 \pi v_{3}^{0}}(\rho * w)(\mathbf{r})-\frac{\alpha}{\pi}(w * u)(\mathbf{r}) \\
+\frac{\gamma_{1}}{\pi} v(\mathbf{r}) w(\mathbf{r})-\frac{\delta}{\pi} \int_{\mathbf{r}^{\prime}} e^{i \mathbf{r} \wedge \mathbf{r}^{\prime}} v\left(\mathbf{r}^{\prime}\right) w\left(\mathbf{r}^{\prime}\right) .
\end{gathered}
$$

Here $\epsilon=\delta=0, \quad \alpha=\beta=1 /\left(2 v_{3}^{1}\right), \quad$ and $\gamma_{1}=4 \gamma_{2}=2 /\left(v_{3}^{0}+v_{3}^{1}\right)$. When the second LL is weakly populated, the Fermi velocity $v_{3}^{1} \ll v_{3}^{0}$ so that $e^{2} / v_{3}^{1} \gg e^{2} / v_{3}^{0}$, implying that the second LL is most susceptible to interactions. It follows that the leading instability is driven by $u$, which couples only these carriers. This can be understood analytically by ignoring all $(f * g)$ terms in Eqs. (8); one then obtains $u(r ; \ell) \approx\left[u(r ; 0)^{-1}\right.$ $\left.-\ell /\left(2 \pi v_{3}^{1}\right)\right]^{-1}$. Due to the form of the second LL wave functions, at the initial conditions $u(r ; 0) \approx g^{\prime}\left(r^{2}-1\right) e^{-r^{2} / 2}$, with $g^{\prime}>0$. The instability occurs at $\ell^{*}=2 \pi v_{3}^{1} / u\left(r^{*} ; 0\right)$, where $r^{*} \neq 0$ maximizes $u(r ; 0)$, driving CDW order formed by con- 
densing $\left\langle c_{3 R}^{1 \dagger} c_{3 L}^{1}\right\rangle$. As shown in Ref. 14, since $u\left(r^{*} \neq 0\right)$ controls the instability, the transverse density becomes Wigner crystalline here unlike in the LLL CDW. We have verified numerically that the $(f * g)$ terms neglected do not modify these conclusions.

Such weak-coupling arguments fail when the second LL is sufficiently dilute since $e^{2} / v_{3}^{1}$ inevitably becomes large as the band empties. To study this regime, we sit at $B_{*}(\theta)$ and fine tune the chemical potential to $\mu_{*}$ in Fig. 1(b). We now have linearly dispersing right/left movers $c_{R / L 3}^{0}$ coupled with quadratically dispersing second LL electrons which we denote by $d_{3}^{1}$. The soft dispersion for the latter renders interactions involving $d_{3}^{1}$ strongly relevant, reflecting the strongcoupling nature of the problem. To proceed, we seek a controlled limit by replacing the second LL kinetic energy $D k_{\|}^{2} \rightarrow D\left|k_{\|}\right|^{1+\epsilon}$ and perform an expansion in $\epsilon$ and the interaction strength in the limit $1 \gg \epsilon \sim e^{2} / v_{3}^{0}{ }^{15}$

Pocket 3 interactions have the same form as Eq. (7) but with $c_{3 R / L}^{1} \rightarrow d^{1}$. The couplings flow as in Eq. (8), where now $\alpha=1 / D, \gamma_{1}=\delta=1 /\left(v_{3}^{0}+D \lambda \epsilon^{\epsilon} e^{-\epsilon \ell}\right), \gamma_{2}=\gamma_{1} \lambda^{\epsilon} e^{-\epsilon \ell}$, and $\beta=0$. At the initial conditions, $\rho$ is the largest coupling, as it involves only LLL carriers, while $w$ is (by far) the smallest since the Dirac LL wave functions involved in this scattering process have little overlap. As a first step, we thus set $w=0$, which decouples the remaining equations. From our earlier analysis, it is clear that both $\rho$ and $v$ then flow off, with the former diverging faster since $\rho$ is larger at the initial conditions. Assuming $u(k, x) \equiv u(\mathbf{r})$ is analytic in $k, x$, we can solve the flows for $u$ very generally by assuming an ansatz $u(r ; \ell)$ $=\sum_{j} f_{j}(\ell) \chi_{j}(r)$. Here $j$ runs over $0,2,4, \ldots$ and $\chi_{j}(r)$ $=e^{-r^{2} / 2} P_{j}(r)$, with $P_{j}(r)$ degree- $j$ polynomials defined so that $\left(\chi_{j}^{*} \chi_{j^{\prime}}\right)(\mathbf{r})=\delta_{j, j^{\prime}} \chi_{j}(r)$. One then obtains decoupled equations for each $f_{j}$ which show that $u(\mathbf{r} ; \ell)$ flows to a finite fixed point provided $f_{j}(0) \geq 0$ for all $j$. As in region $\mathrm{I}, u(\mathbf{r} ; \ell=0)$ $\approx g^{\prime}\left(r^{2}-1\right) e^{-r^{2} / 2}=2 \pi^{2} g^{\prime} \chi_{2}(r)$, with $g^{\prime}>0$, which indeed remains finite under renormalization.

To include $w \neq 0$, we study the FRG equations numerically. Here we find that $w$ further enhances the instability in $\rho$ and also generates additional nonzero components of $\chi_{j}$ in $u$, causing this function to flow off in tandem with $w$. In contrast, $v$ is only weakly affected by $w$ and remains unimportant. Because $\rho$ was already unstable when $w=0$, this function becomes of order 1 first, driving the LLL CDW order as found in region II. Soon after, $u$ becomes of order 1 .
Consequently, as the chemical potential increases, strong interactions encoded in this coupling Wigner crystallize the dilute second LL carriers. While this is reasonable, we caution that ruling out the scenario where $u$ provides the leading instability is difficult in the physical $\epsilon=1$ case. In particular, the $w=0$ fixed point for $u$ found above could move to strong coupling with $\epsilon=1$, which would imply a $u$-driven Wigner crystal instability followed by the LLL CDW order.

Putting our results together, we propose the minimal phase diagram shown in Fig. 1(c). We speculate that experiments of Ref. 3 may be conducted above the critical temperature for the CDW phases in regions I and II but below that for the Wigner crystal phase near the boundary. Firstorder transitions from the latter would be consistent with the observed hysteresis. However, two transitions ought to occur here, whereas experiments see only one. This issue can be resolved by invoking disorder, which will be particularly important in the low-density region of the Wigner crystal phase close to region II. The transition on that side is expected to be smeared by disorder pinning of the localized states, which should be addressed in more detail. Future experiments, particularly, nonlinear transport and x-ray scattering studies to search for signs of CDW and Wigner crystal order, should provide valuable clues as to the true nature of this transition.

The Hall- and Nernst-effect puzzles in trigonal fields $B>9 \mathrm{~T}^{2}$ are difficult to resolve at weak coupling. Here, hole-hole pairing drives the leading CDW instability, which is not expected to recover these anomalies. While surface states ${ }^{16}$ should be seriously considered as emphasized in Ref. 3 , we believe a more exotic origin is not inconceivable. Interactions between the LLL holes are not weak, as $e^{2} / v_{h}^{0}$ $\sim 24$, well outside of the range where weak coupling is expected to be reliable. In contrast, LLL interactions, while not weak, are several times smaller: $e^{2} / v_{e}^{0} \sim 5$. Although screening should reduce these somewhat, the problem warrants studying from a strong-coupling standpoint, which presents an exciting research direction. ${ }^{17}$

It is a pleasure to acknowledge illuminating discussions with K. Behnia, Lu Li, O. Motrunich, N. P. Ong, and G. Refael, as well as the hospitality of the KITP where this work was initiated. We also acknowledge support from the Lee A. DuBridge Foundation (J.A.) and the Packard Foundation and National Science Foundation through Grants No. DMR-0804564 and No. PHY05-51164 (L.B.).

\footnotetext{
${ }^{1}$ V. S. Edel'man, Adv. Phys. 25, 555 (1976).

${ }^{2}$ K. Behnia et al., Science 317, 1729 (2007).

${ }^{3}$ L. Li et al., Science 321, 547 (2008).

${ }^{4}$ A. Banerjee et al., Phys. Rev. B 78, 161103(R) (2008).

${ }^{5}$ G. E. Smith et al., Phys. Rev. 135, A1118 (1964).

${ }^{6}$ M. H. Cohen et al., Philos. Mag. 5, 115 (1960).

${ }^{7}$ V. M. Yakovenko, Phys. Rev. B 47, 8851 (1993).

${ }^{8}$ A. A. Abrikosov, J. Low Temp. Phys. 10, 3 (1973).

${ }^{9}$ Y. Liu et al., Phys. Rev. B 52, 1566 (1995).

${ }^{10}$ S. G. Bompadre et al., Phys. Rev. B 64, 073103 (2001).
}

${ }^{11}$ The data of Ref. 10 appear consistent with the hole Zeeman splitting being $2 \pm 0.16$ times the cyclotron energy. We use the lower sign, which gives $g_{h}=54$, since the hole quantum limit then occurs closer to $9 \mathrm{~T}$ as observed experimentally.

${ }^{12}$ B. I. Halperin and T. M. Rice, Rev. Mod. Phys. 40, 755 (1968).

${ }^{13}$ R. Shankar, Rev. Mod. Phys. 66, 129 (1994).

${ }^{14}$ S. W. Tsai et al., Phys. Rev. B 65, 241102(R) (2002).

${ }^{15}$ L. Balents and M. P. A. Fisher, Phys. Rev. B 53, 12133 (1996).

${ }^{16}$ T. E. Huber et al., Phys. Rev. B 79, 201304(R) (2009).

${ }^{17}$ F. J. Burnell et al., Phys. Rev. B 79, 155310 (2009). 